\title{
Evaluation of the Ability to Apply Near-Infrared Spectroscopy on Direct Assay of Acyclovir in Tablets
}

\author{
Le Dinh Chi ${ }^{1}$, Tran Viet Hung ${ }^{2}$, Bui Van Trung ${ }^{3}$ and Doan Cao Son ${ }^{4}$ \\ 1. The Department of Analytical Chemistry and Toxicology, Hanoi University of Pharmacy, Hanoi 110403, Vietnam \\ 2. Director, Institute of Drug Quality Control Ho Chi Minh City, Ho Chi Minh City 712161, Vietnam \\ 3. The Laboratory of Physical Measurements, National Institute of Drug Quality Control, Hanoi 110116, Vietnam \\ 4. Director, National Institute of Drug Quality Control, Hanoi 110116, Vietnam
}

\begin{abstract}
This paper aimed at developing a simple and fast approach using chemometrics processing for direct assay of acyclovir in tablets by NIR (near infrared) spectroscopy in diffuse reflectance mode. In making trials with 5 different tablet matrices, the experimental results showed that regardless the matrix variation, it was always possible to construct a quantitative model with suitable linear range, accuracy and precision for direct assay of acyclovir in tablet from NIR spectra. Therefore, the approach used in this study was suitable for on-site fast assay of APIs in tablets during manufacturing process or in post-marketing surveillance of drug quality.
\end{abstract}

Key word: Near-infrared spectroscopy, chemometrics, acyclovir, tablet, partial least square regression, direct assay.

\section{Introduction}

Pharmaceutical markets in developing countries are characterized by a high diversity in terms of active principles and dosage forms, as well as in origin of pharmaceutical products. This heterogeneity results in the necessity of extensive post-marketing surveillance from authorities to assure the safety and efficacy of drugs. And such highly competitive markets force manufacturers look for more efficient and economic ways to improve the quality of their products and reduces damages from substandard products.

Conventional methods for drug quality control such as those in pharmacopoeia (USP (United States Pharmacopoeia) 38, BP (British Pharmacopoeia) 2017; etc.) and in-house standards are reliable but time consuming, sample destroying and needed to be done in laboratories suitably equipped for this purpose [1, 2]. This means a considerable delay between sampling and obtaining analysis results, and sometimes when a drug sample is detected as substandard, it is already too late

Corresponding author: Doan Cao Son, Ph.D., assoc. prof., Analytical chemistry and drug quality control. to prevent the whole batch it belonged to from reaching patients or to avoid economic damages for manufacturers. Hence, fast screening of drug samples in market for an effective post-marketing surveillance is still a great challenge for the authorities. In the other hand, manufacturers nowadays also want to ensure the quality of their products down to each individual dosage unit, and in the same time economizing the cost and workload for QC (quality control) activities.

In contrast to conventional methods, fast, non-destructive spectroscopic analytical techniques are suitable for quick on-site analysis of drug products [3, 4]. They can be used for market surveillance screening or in-process control at manufacturing facilities. By providing instantaneous results, they permit a closer quality monitoring of drug products in the market as well as during and at the end of manufacturing process. Moreover, thanks to these screening tests, sampling was necessary only for products giving suspected screening results $[5,6]$. Therefore, sampling processes become more selective and effective, and in the same time the number of samples to be analyzed in QC laboratories increase. This results in reducing the 
burden and the cost for QC laboratories.

With advances in instrumentation and software, NIR (near infrared) spectroscopy has been known as a rapid, non-destructive analysis method [7, 8]. Diffuse reflectance mode combines with multivariate analysis may be used for qualitative and quantitative without sample preparation [9-11]. NIR methods have been developed to monitor many phases of the manufacturing process, from the arrival of bulk raw material to the inspection of tablets for final release and controlling the drug quality in pharmaceutical market [12-14]. Especially, handheld NIR instrument can be used for on-site screening for counterfeit drugs [15, 16]. Therefore, it may be a sound technical solution for the above-mentioned challenges.

Acyclovir

(Fig.

1 ,

2-Amino-1,9-dihydro-9-((2-hydroxyethoxy) methyl)

-3H-purin-6-one) is an antiviral drug used to treat infections caused by herpes viruses, such as genital herpes, cold sores, shingles, and chicken pox [17]. In many countries, such infections are still a significant health issue, therefore anti-herpes drugs, including products containing acyclovir, occupy an important market share with important economic profit. The latter aspect makes acyclovir a potential choice for fabrication of counterfeit drugs as well as a priority for sampling in post marketing surveillance. Validated analysis methods for assay of acyclovir in dosage forms are available in USP using HPLC (high performance liquid chromatography) (for capsule, powder for injection, ointment, oral suspension, tablet) [1], or in BP using ultraviolet-visible (UV-Vis) spectroscopy (for cream, eye ointment, infusion, tablet, dispersible tablet), and fluorescent spectroscopy (for oral suspension) [2]. However, so far, no official NIR method for fast assay of acyclovir in dosage forms has yet been provided in any pharmacopoeia, and publication regarding the use of NIR spectroscopy for assay of acyclovir is still rare.

This study evaluates ability to use NIR spectroscopy for quantitative analysis of acyclovir by assessing the

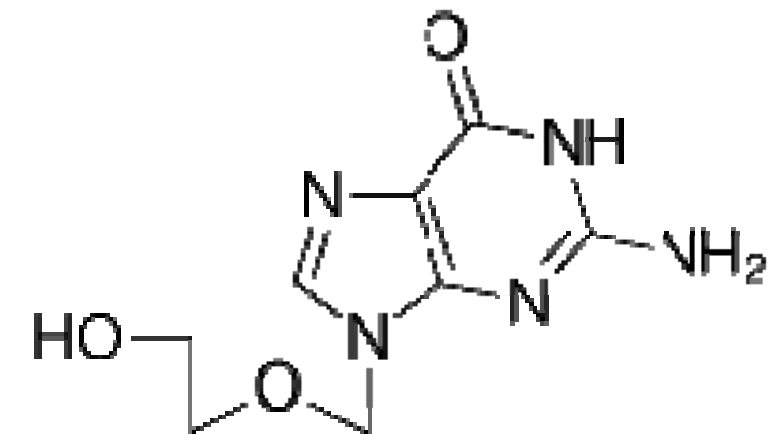

Fig. 1 Structural formulation of acyclovir.

suitability and reliability of this technique in assaying acyclovir from different tablet matrices.

\section{Material and Methods}

\subsection{Material}

Acyclovir, active principle for pharmaceutical usage, was provided by National Institute of Drug Quality Control, (batch No.: 0105137, containing 98.87\% acyclovir, water content: 3.99\%).

Excipients for pharmaceutical usage, all meet the requirements of pharmacopoeia of manufacturing countries: starch, wheat starch, lactose, sodium lauryl sulfate, ethanol (all manufactured in Vietnam), tabletose, sodium croscarmellose, dicalcium hydrophosphate, avicel, aerosil, talc, magnesium stearate, sodium starch glycolate, PVP-K30, hydroxypropyl methylcellulose (all manufactured in China).

Information regarding the theoretical tablet weights of several commercial tablets showed in Table 1 was graciously provided by respective manufacturers upon request from authors of this paper (Synmedic Laboratories (India) for ACIRAX-400, DHG Pharma (Vietnam) for MEDSKIN ACYCLOVIR 200, Mediplantex (Vietnam) for ACYCLOVIR $200 \mathrm{mg}$, and STADA-VN (Vietnam) for Acyclovir STADA 400 $\mathrm{mg}$ )

\subsection{Equipment and Software}

Analytical balance AB-204S with readability of 0.1 mg (Mettler Toledo, Ohio, USA). 
Table 1 Content of acyclovir in several commercial tablets.

\begin{tabular}{lllll}
\hline No. & Commercial products & $\begin{array}{l}\text { Weight of tablet } \\
(\mathrm{mg})\end{array}$ & $\begin{array}{l}\text { Amount of acyclovir in label claim } \\
(\mathrm{mg})\end{array}$ & $\begin{array}{l}\text { Content of acyclovir } \\
(\% \mathrm{w} / \mathrm{w})\end{array}$ \\
\hline 1 & ACIRAX-400 & 620.0 & 400.0 & 64.5 \\
2 & MEDSKIN ACYCLOVIR 200 & 327.0 & 200.0 & 61.2 \\
3 & ACYCLOVIR 200 mg & 335.0 & 200.0 & 59.7 \\
4 & Acyclovir STADA 400 mg & 575.0 & 400.0 & 69.6 \\
\hline
\end{tabular}

Tablet compression machine: Manual Hydraulic Press (Specac, Pennsylvania, USA).

Near Infrared Spectrometer IS50NIR (Thermo Fischer Scientific, Massachusetts, USA) installed with OMNIC Spectra Software version 9.2 for spectral acquisition (Thermo Fischer Scientific, Massachusetts, USA).

TQ Analyst ${ }^{\mathrm{TM}}$ Pro. Edition Software version 9.2 for data processing (Thermo Fischer Scientific, Massachusetts, USA).

\subsection{Experimental Samples}

To evaluate the ability of using NIR spectroscopy for direct assay of acyclovir in tablet, experimental tablets were manufactured by using 5 different formulations to provide 5 different matrices. Components of each matrix were constructed based on the common tablet excipients with suitable amounts, they include diluents, binders, disintegrants, lubricants, glidants and miscellanies. In each placebo matrix, experimental tablets were made with several levels of acyclovir content $(\%, w / w)$, in which the central level (target content) was selected to be similar to that in commercial tablets, and other levels were established around this central level. To determine the target content, the percentage $(\%, w / w)$ of acyclovir in different commercial tablets was investigated, showing that the normal content of acyclovir in tablet fell within $60 \%$ and $70 \%(\mathrm{w} / \mathrm{w})$ (see Table 1 ). Therefore, the target contents of acyclovir in experimental tablet were fixed at $60 \%$ and $70 \%$. And for each matrix, besides target contents, experimental tablets were made with acyclovir levels at other levels to cover the range from 5 to $90 \%(w / w)$ (at $5 \%, 10 \%, 20 \%, 25 \%, 50 \%, 60 \%$, $70 \%, 80 \%$, and $90 \%$ ), and presented in sample formulation section, in order to evaluate the linearity between content of acyclovir in tablet and NIR spectral responses and so to investigate the ability for quantitative applications.

The principle of quantitative assay with NIR bases on the determination of API ratio $(\%, w / w)$ in drug matrix [12, 18]. So, in theory, NIR does not need an exact weight of sample. In contrast, in NIR analysis, it is necessary to prevent the effect of outside light, especially diffuse reflectance mode. Therefore, the thickness and surface size of sample are important factors. Sample thickness must be enough to ensure that light cannot travel through sample and the surface size (thus diameter) of sample must be large enough to receive the whole NIR beam. As a consequence, every experimental tablet had overall weight about $500 \mathrm{mg}$ but the content $(\%, w / w)$ of acyclovir varied within the previously mentioned range. Experimental tablets are made by direct compression after homogenous mixing of formulation ingredients.

\subsection{Method}

\subsubsection{Spectral Acquisition}

NIR spectra are acquired by diffusing reflectance tool over the range from $4,000 \mathrm{~cm}^{-1}$ to $10,000 \mathrm{~cm}^{-1}$. Each spectrum is the average of 32 scans data resolution of $4 \mathrm{~cm}^{-1}$. Before experiment, background was recorded and rescanned after 2 hours. All experimental tablets received whole NIR beam spot during scanning.

\subsubsection{Data Processing}

Spectra recorded were divided into subsets for processing. Each subset includes 50 spectra of 50 tablets of each matrix formulation. In each subset, spectra were divided randomly into two groups, a 
group of calibration and a group of prediction. The number of spectra in each group was also selected randomly, but a subset contained not more than 43 calibration spectra and not more than 13 validation spectra.

Quantitative models constructed from raw spectra by using TQ Analyst software version 9.2 following three steps: first derivative; spectral ranges selected manually in the range from $4,000 \mathrm{~cm}^{-1}$ to $10,000 \mathrm{~cm}^{-1}$, in each range, baseline type is two points and average in range; lastly, PLS multivariate regression applied for all the data in each spectral subset.

\section{Results and Discussion}

\subsection{Results}

\subsubsection{Sample Formulation}

Five different formulations using common excipients for tablets were established and used for manufacturing experimental tablet containing different levels (\%,w/w) of acyclovir as presented in Table 2. These tablets were used for establishing quantitative model to assay acyclovir in tablets.

3.1.2 Establishing Calibration Models for Assay of Acyclovir from NIR Spectra

Quantitative models for assay of acyclovir were constructed from data subsets, each consisting of 50 spectra corresponds to 10 content levels of acyclovir (or concentration point), and each concentration point consists of 5 spectra obtained from 5 experimental tablets having the same content level of acyclovir. Each subset corresponds to a specific tablet matrix. Raw spectra of 50 tablets in formulation 2 were presented in Fig. 2.

It was clear that spectra showed important tablet-to-tablet variations even at the same concentration point. From quantitative view, it is difficult to find directly any correlation between concentration of acyclovir in tablet and spectral response on the raw spectra. Therefore, a spectral processing was used to construct quantitative model for assay of acyclovir. In the first step, raw spectra were transformed into 1st derivative spectra. The 1st derivative spectra made it easier to find the ranges where proportional correlation was most evident between acyclovir content and spectral intensity (see Fig. 3).

In the second step, by visually comparing 1st derivative spectra corresponding to different concentrations of acyclovir in experimental tablets, the most relevant ranges from 1st derivative spectra of each subset were selected manually for final processing. The manual selection of spectral ranges was done by finding visually the correlation between variation of spectral intensity and that of acyclovir concentration (see Fig. 4). Software can help select from 1st derivative the spectral zones with best linearity. These zones gave fittest relationship between response of acyclovir and excipient matrix, but they are not always

Table 2 Acyclovir content levels (\%, w/w) for each matrix formulation.

\begin{tabular}{llllll}
\hline & \multicolumn{5}{c}{ Matrices } \\
\cline { 2 - 6 } & Formulation 1 & Formulation 2 & Formulation 3 & Formulation 4 & Formulation 5 \\
\hline & 5.35 & 5.08 & 4.90 & 5.02 & 4.88 \\
& 10.16 & 9.88 & 10.09 & 9.93 & 10.05 \\
& 15.42 & 15.11 & 14.85 & 15.00 & 14.96 \\
Acyclovir & 19.96 & 20.10 & 20.05 & 19.98 & 20.23 \\
content level & 26.60 & 25.23 & 25.09 & 25.15 & 25.23 \\
$(\%$, w/w) & 39.64 & 39.91 & 40.03 & 40.09 & 39.82 \\
& 49.32 & 50.35 & 60.64 & 50.02 & 49.68 \\
& 59.85 & 60.17 & 70.37 & 59.94 & 59.60 \\
& 70.25 & 70.00 & 80.07 & 70.05 & 69.62 \\
\hline
\end{tabular}




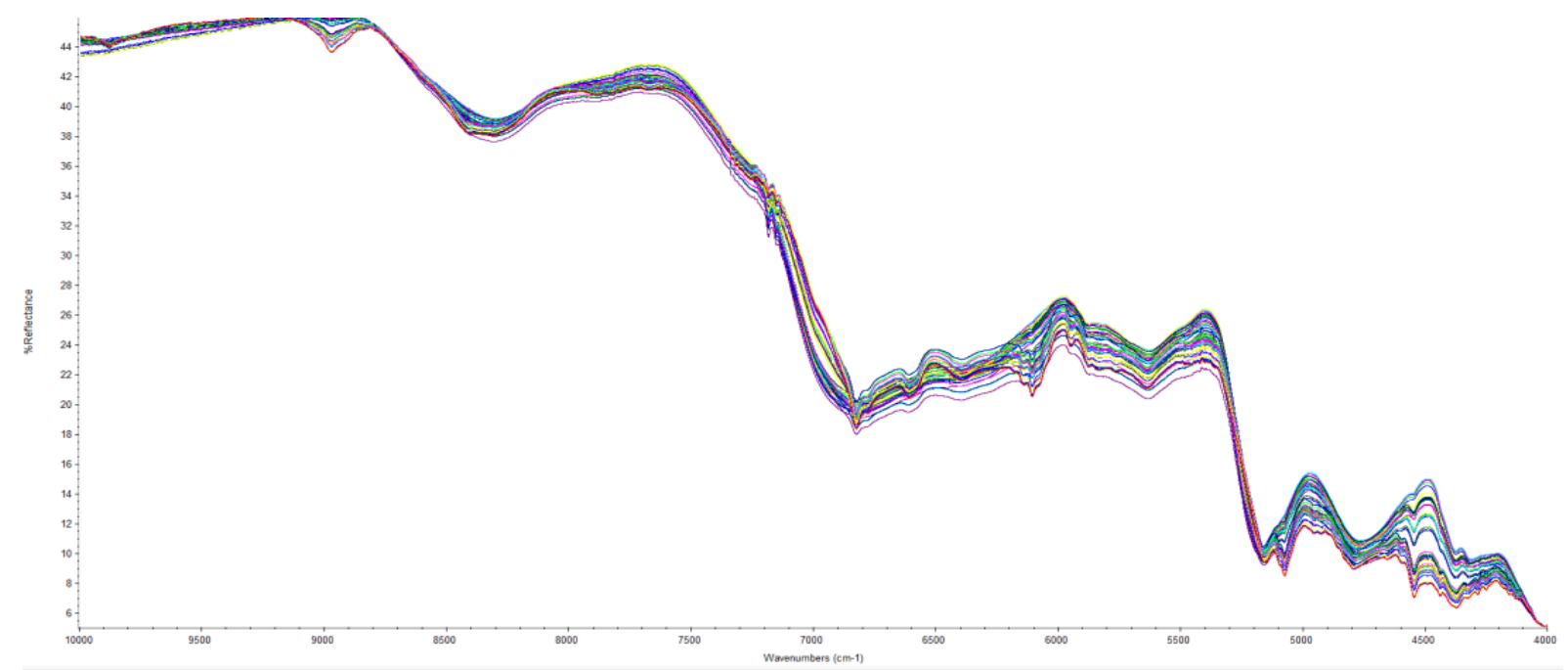

Fig. 2 Raw NIR spectrum of acyclovir in tablets formulation 2.

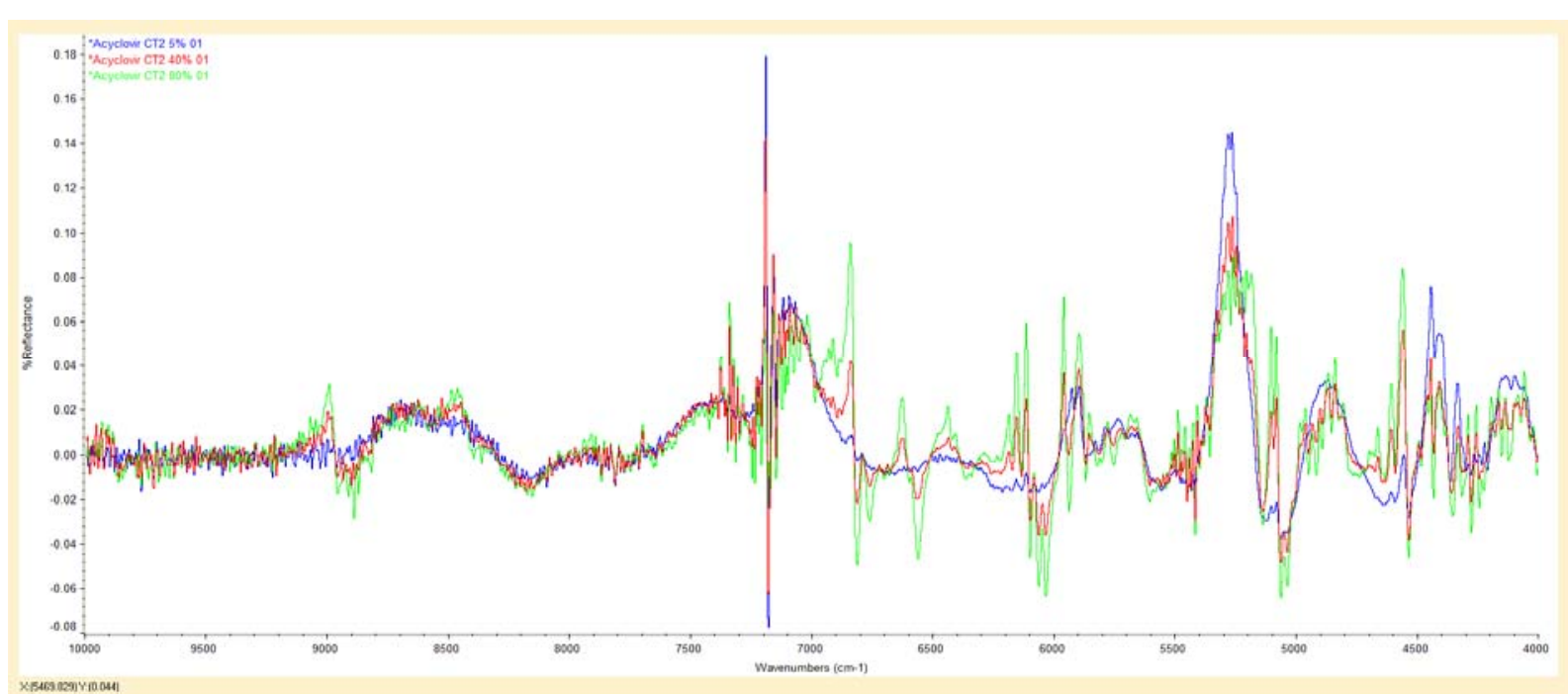

Fig. 3 1st derivative spectra of acyclovir tablets in formulation 2.

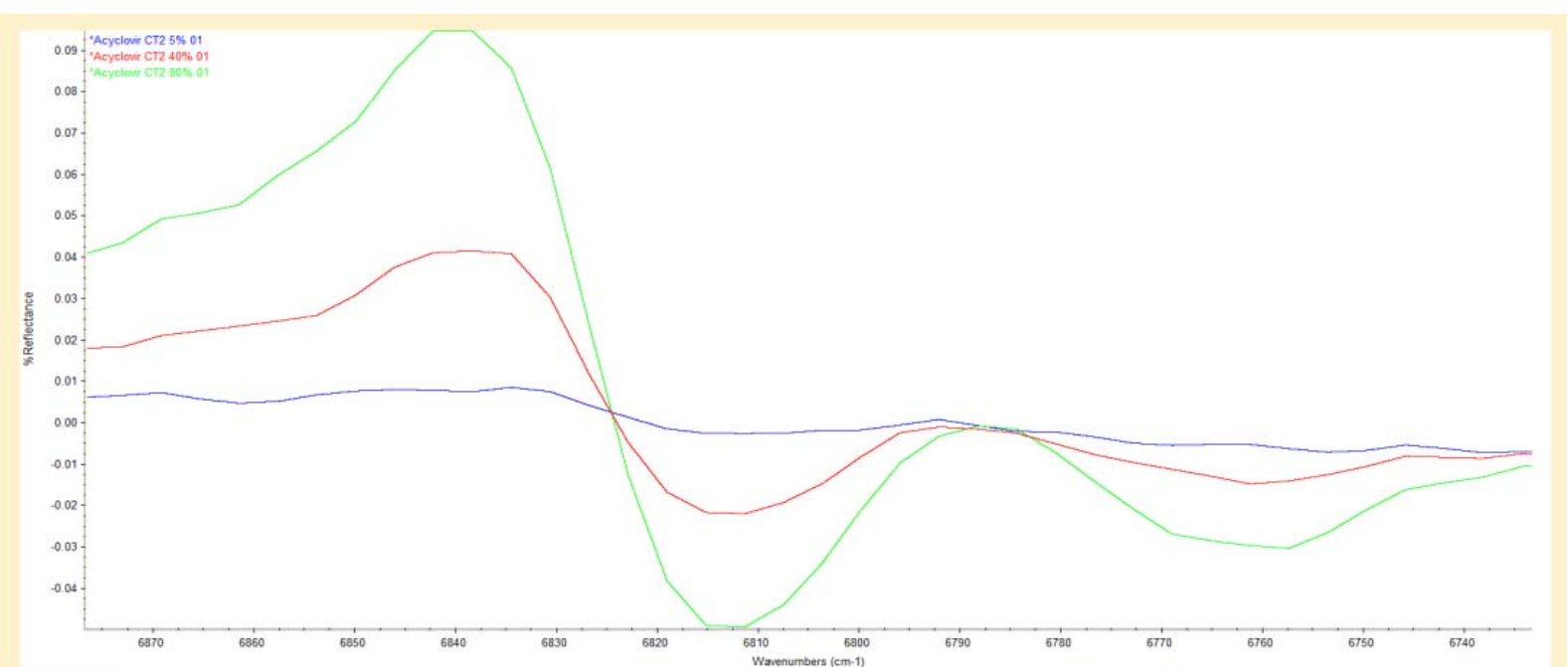

Fig. 4 A specific range was selected in full range of 1st derivative spectra of acyclovir tablets in formulation 2 for construction of quantitative model. 
zones providing best responses among the compositions of analyzing sample. For example, in case the selected spectral zone gave good linearity but the intensity of response was low, quantitative analysis at this zone may lead to significant error due to measure-to-measure variation and instability of NIR instrument. Hence, the selection of relevant spectral ranges must be done manually.

In the third step, PLS at multiplicative signal correction was applied on the relevant ranges of spectra selected in second step. Within a subset, calibration spectra randomly selected from the beginning by software provided data for building calibration model of assay method, and the remaining spectra were used for validation of model. A typical result obtained from a subset was demonstrated in Fig. 5. In this case, 41 spectra were used for built calibration method and 9 spectra were used for validation of method. And 4 factors were the best selection for this model because it suggested the best ratio of RMSEC and RMSEP as well as the best correlation coefficient for both calibration and validation with 1.000 and 0.9999 correspondently.

Similar processes were applied for other model of acyclovir in other matrices, the calibration result was listed in Table 3.

The linear model provided the content Y (\%, w/w) of acyclovir in sample. From the $\mathrm{Y}$ value, the predicted amount $\mathrm{X}$ of acyclovir in sample was calculated as followed:

$$
\mathrm{X}=\mathrm{Y} \times \mathrm{m}
$$

where, $\mathrm{X}$ is the amount of acyclovir in tablet sample (mg); Y is the content (w/w) calculated by linear model; $\mathrm{m}$ is the weight of tablet sample (mg).

To determine the acyclovir amount in a tablet, it is necessary only to record NIR spectrum of sample and apply into corresponding model.

\subsubsection{Validation of Calibration Models}

Each quantitative model that was constructed was validated in term of linearity, accuracy and precision.

(a) Linearity

As presented in Table 3, all quantitative models showed good linearity between the acyclovir amounts $\mathrm{X}$ and the model response $\mathrm{Y}$, which was similar to the degree of linearity usually observed and required with conventional method for assay of API in pharmaceutical dosage forms.

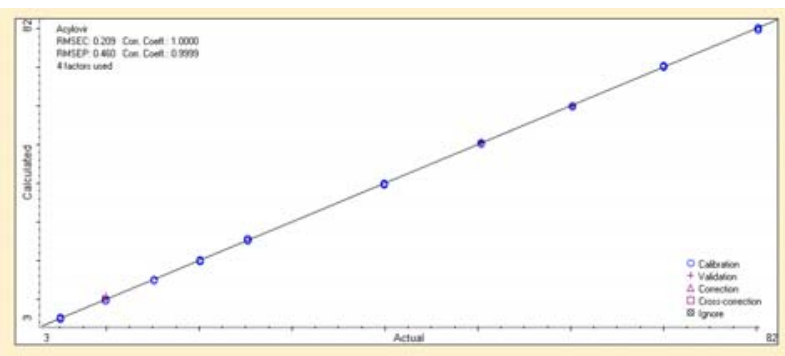

(A)

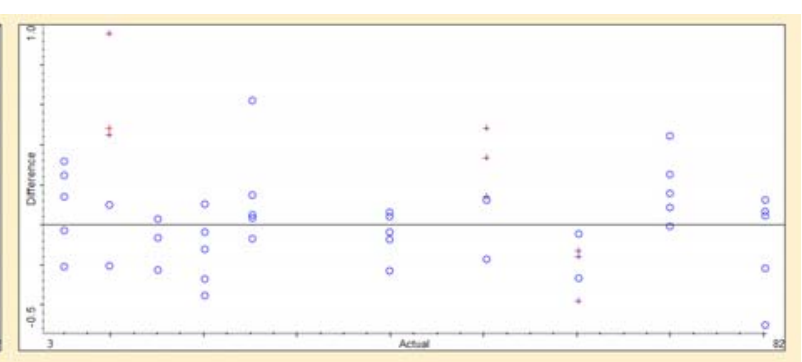

(B)

Fig. 5 Graph of the calibration models describes the correlation between content of acyclovir in formulation 2 tablets and their NIR spectra (A-a linear curve was constructed from 10 points of concentration and their 1st derivative spectra, B-concentrate tolerance of calibration and validation around established curve).

Table 3 Result obtained for linear quantitative model of calibration and validation acyclovir in tablets.

\begin{tabular}{|c|c|c|c|c|c|c|}
\hline \multirow{2}{*}{ No. } & \multirow{2}{*}{$\begin{array}{l}\text { Formulation } \\
\text { name }\end{array}$} & \multirow{2}{*}{ Factor number } & \multicolumn{2}{|c|}{ Calibration } & \multicolumn{2}{|c|}{ Validation } \\
\hline & & & $\mathrm{R}$ & RMSEC & $\mathrm{R}$ & RMSEP \\
\hline 1 & Formulation 1 & 4 & 0.9998 & 0.502 & 0.9996 & 0.745 \\
\hline 2 & Formulation 2 & 4 & 1.0000 & 0.209 & 0.9999 & 0.460 \\
\hline 3 & Formulation 3 & 4 & 0.9999 & 0.314 & 0.9997 & 0.576 \\
\hline 4 & Formulation 4 & 4 & 1.0000 & 0.217 & 0.9995 & 0.467 \\
\hline 5 & Formulation 5 & 4 & 0.9999 & 0.317 & 0.9998 & 0.488 \\
\hline
\end{tabular}


(b) Accuracy and precision

To validate the precision and accuracy of quantitative models, for each formulation of matrix, the assay of acyclovir was done with experimental tablets containing 3 different contents (\%,w/w) of acyclovir, including the target concentration as mentioned in 2.3. In terms of precision, for all matrices, the variation of acyclovir content calculated from models was low at each real content level (RSD $\leq 1.1 \%$, see Table 4). Similarly, in term of accuracy, the results of acyclovir concentrations calculated from models showed good recoveries (99.4-100.8\%, see Table 4), within the normal recovery limits from 98-102\% generally required for assay method of API in pharmaceutical dosage forms using conventional techniques (HPLC, UV-Vis spectroscopy, etc.) [19].

\subsection{Discussion}

The use of chemometrics has enabled the use of NIR spectroscopy for quantitative purpose as being showed in this study. The processing of original spectral data by derivation and applying PLS has resulted in quantitative models which can determine precisely and accurately the concentration of acyclovir $(\%, w / w)$ in tablets. The effectiveness and feasibility of this approach were confirmed from the possibility of constructing such models for 5 different formulation matrices. The data processing helped simultaneously resolve the most important hindrances regarding the use of NIR spectroscopy in quantitative application. In the one hand, it solved the problem of unstable optical path length in diffuse reflectance mode, thus enabling NIR to become a direct analytical method, with simple sample preparation and fast analysis. In the other hand, chemometrics transformed overlap and mixed raw data into a suitable form rich information data, more relevant for intended application. Correlation between spectral data and sample characteristic become clearer and more visually detectable, made it easy to find relation between the content of analyze and spectral information and select manually the best conditions for developing qualitative model for assay of analyze. Moreover, chemometrics based on statistic algorithms, therefore the establishment of quantitative methods and, consequently the obtained results, were insured about their impartiality.

NIR spectroscopy combining diffuse reflectance is a direct and nondestructive method analyzing samples in their original state. It reduces error coming from sample preparation process. The reliability of API

Table 4 Result of accuracy and precision validation.

\begin{tabular}{|c|c|c|c|c|}
\hline $\begin{array}{l}\text { Formulation } \\
\text { name }\end{array}$ & $\begin{array}{l}\text { Real content of acyclovir } \\
(\%, w / w)\end{array}$ & $\begin{array}{l}\text { Predicted content of acyclovir } \\
(\%, w / w)\end{array}$ & $\begin{array}{l}\text { RSD of } \\
\text { prediction (\%) }\end{array}$ & $\begin{array}{l}\text { Recovery } \\
(\%)\end{array}$ \\
\hline \multirow{3}{*}{ Formulation 1} & 49.32 & 49.09 & 0.5 & 99.5 \\
\hline & 64.82 & 64.57 & 0.8 & 99.6 \\
\hline & 70.25 & 70.82 & 0.3 & 100.8 \\
\hline \multirow{3}{*}{ Formulation 2} & 50.35 & 50.49 & 1.0 & 100.3 \\
\hline & 61.32 & 61.18 & 0.8 & 99.8 \\
\hline & 70.14 & 70.34 & 0.4 & 100.3 \\
\hline \multirow{3}{*}{ Formulation 3} & 49.32 & 49.09 & 0.5 & 99.5 \\
\hline & 59.85 & 59.60 & 0.9 & 99.6 \\
\hline & 70.25 & 70.82 & 0.3 & 100.8 \\
\hline \multirow{3}{*}{ Formulation 4} & 60.25 & 60.00 & 0.9 & 99.6 \\
\hline & 69.32 & 69.89 & 0.3 & 100.8 \\
\hline & 80.07 & 80.03 & 0.4 & 100.0 \\
\hline \multirow{3}{*}{ Formulation 5} & 49.48 & 49.80 & 0.6 & 100.6 \\
\hline & 64.88 & 64.46 & 0.8 & 99.4 \\
\hline & 69.95 & 69.71 & 0.7 & 99.7 \\
\hline
\end{tabular}


assay by this technique was demonstrated as equal to that of analysis techniques commonly used in drug quality control like HPLC or UV-Vis spectroscopy in terms of precision, accuracy, as showed in the validation results of this study. In addition, once established, the assay method using NIR spectroscopy is fast and easy to use, suitable for on-site immediate analysis and do not use any toxic solvent or chemical, ideal for "green chemistry".

The quantitative models in this study were established for 5 types of experimental tablets with 5 different formulations. Each of these formulations was established from ingredient compositions commonly used in manufacturing of tablets. The fact that quantitative models for assay of acyclovir were feasible from NIR spectral information regardless the variation of matrix composition showed that the data processing proposed in this study can be used as a common chemometrics approach for almost any other matrix and other API. Furthermore, this approach can be used for quantitative determination of not only API but any composition of interest in a formulation.

\section{Conclusions}

According to this study results, NIR spectroscopy when using diffuse reflectance mode combining with chemometric approach in data processing has been proved as an efficient quantitative method, especially for tablet form. For assay of a composition in tablet like acyclovir, this method satisfied the requirements in linearity, accuracy and precision of some referent method which are widely used in pharmaceutical industry as well as in control drug quality. The NIR quantitative models for assay of acyclovir in tablets were established using experimental tablets containing 5 different formulation matrices representing real acyclovir tablet matrices in the market. They were constructed based on the respondent of different acyclovir concentrations in a random specific placebo matrix, and therefore did not depend on any specific matrix. Therefore, the processing model that used to establish method can apply for any other tablet formulations. In conclusion, the combination of NIR spectroscopy and multivariate analysis has proved to be a sound approach for developing fast and reliable quantitative methods, especially for assay of API in tablet form. This kind of NIR-based method can be widely applied for drug quality control in market as well as in manufacturing process. From experiences obtained with this study, NIR assay method work best with a fixed sample matrix. Therefore, a logical next step after this study is adapting the same approach to some real commercial product with stable manufacturing process and formulation to develop a fast alternative method for assay of API besides the existing conventional method.

\section{References}

[1] US Pharmaceutical Convention Inc. 2015. United States Pharmacopoeia. 38 ed., Vols. I, II, III, Rockville.

[2] Her Majesty's Stationary Office. 2017. British Pharmacopoeia 2017. Vols. I, II, III, London.

[3] Amira, T., Peczalski, R., and Zagrouba, F. 2016. “On-line Monitoring of Vacuum Drying of Theophylline Using NIR Spectroscopy: Solid-State Transitions, Water Content and Semi-Empirical Modeling." Drug Development and Industrial Pharmacy 42 (11): 1825-32.

[4] Klara Dégardin, Aurélie Guillemain, and Yves Roggo. 2017. "Comprehensive Study of a Handheld Raman Spectrometer for the Analysis of Counterfeits of Solid-Dosage Form Medicines.” Journal of Spectroscopy 2017 (4): 1-13.

[5] Leia, Y., Luoa, Z. Y., and Hub, C. Q. 2008. "Rapidly Screening Counterfeit Drugs Using near Infrared Spectroscopy: Combining Qualitative Analysis with Quantitative Analysis to Increase Effectiveness.” Journal of Near Infrared Spectroscopy 16 (3): 349-55.

[6] Neuberger, S., and Neusüß, C. 2015. "Determination of Counterfeit Medicines by Raman Spectroscopy: Systematic Study Based on a Large Set of Model Tablets.” Journal of Pharmaceutical and Biomedical Analysis 112: 70-8.

[7] Da Silva, N. C., Honorato, R. S., Pimentel, M. F., Garriques, S., Cervera, M. L., and de la Guardia, M. 2015. "Near Infrared Spectroscopy Detection and Quantification of Herbal Medicines.” Journal of Forensic Sciences 60 (5): 1199-205.

[8] Tatavarti, A. S., Fahmy, R., Wu, H., et al. 2005. 
“Assessment of NIR Spectroscopy for Nondestructive Analysis of Physical and Chemical Attributes of Sulfamethazine Bolus Dosage Forms.” AAPS Pharm Sci. Tech. 6 (1): 91-9.

[9] Dong, J. X., Zhou, W. B., Feng, Y. C., Song, D. Q., and Hu, C. Q. 2000. "Quantitative Calibration Models for the Determination of Azithromycin and Decladinosylazithromycin in Azithromycin Injection Powders Using Diffuse Reflectance Near Infrared Spectroscopy.” Journal of Near Infrared Spectroscopy 419 (1): 45-54.

[10] Hecht, H. G. 1980. "Quantitative Analysis of Powder Mixtures by Diffuse.” Applied Spectroscopy 34 (2): 161-4.

[11] Spragg, R. A. 1984. "A Rapid Sample Preparation Technique for Diffuse Reflectance Measurements.” Applied Spectroscopy 38: 604-5.

[12] Camps, C., Toussirot, M., Quennoz, M., and Simonnet, X. 2011. "Determination of Artemisinin and Moisture Content of Artemisia Annua L. Dry Powder Using a Hand-Held Near Infrared Spectroscopy Device.” Journal of Near Infrared Spectroscopy 19: 191-8.

[13] De Oliveira Neves, A. C., Soares, G. M., de Morais, S. C., da Costa, F. S., Porto, D. L., and de Lima, K. M. 2012. "Dissolution Testing of Isoniazid, Rifampicin, Pyrazinamide and Ethambutol Tablets Using Near-Infrared Spectroscopy (NIRS) and Multivariate Calibration.” Journal of Pharmaceutical and Biomedical Analysis 57: 115-9.
[14] Da Silva, V. H., Goncalves, J. L., Vasconcelos, F., Pimentel, M. F., and Pereira, C. F. 2015. "Quantitative Analysis of Mebendazole Polymorphs in pharmaceutical Raw Materials Using Nearinfrared Spectroscopy.” Journal of Pharmaceutical and Biomedical Analysis 115: 587-93.

[15] Vredenbregt, M. J., Blok-Tip, L., Hoogerbrugge, R., Barends, D. M., and de Kaste, D. 2006. "Screening Suspected Counterfeit Viagra and Imitations of Viagra with Near-Infrared Spectroscopy." Journal of Pharmaceutical and Biomedical Analysis 40 (4): 840-9.

[16] Lu, F., Weng, X. X., Chai, Y. F., Yang, Y. J., Yu, Y. J., and Duan, G. L. 2013. “A Novel Identification System for Counterfeit Drugs Based on Portable Raman Spectroscopy." Chemometrics and Intelligent Laboratory Systems 127: 63-9.

[17] Ajima, U., and Onah, J. O. 2015. "Spectrophotometric Determination of Acyclovir after Its Reaction with Ninhydrin and Ascorbic Acid." Journal of Applied Pharmaceutical Science 5 (4): 65-9.

[18] Laasonen, M., Tuulikki, H.-P., Christine, S., Markku, R., and Heikki, V. 2003. "Development and Validation of a Near-Infrared Method for the Quantitation of Caffeine in Intact Single Tablets.” Analytical Chemistry 75 (4): 754-60.

[19] ICH Harmonised Tripartite Guideline. 2005. "Validation of Analytical Procedures: Text and Methodology.” Q2 (R1). 